\title{
Discovery of lipotoxicity-sensitive transcription factors in non-alcoholic steatohepatitis
}

Joaquín Pérez-Schindler, ${ }^{1, *}$, Elyzabeth Vargas-Fernández ${ }^{1}$, Bettina Karrer-Cardel ${ }^{1}$, Danilo

Ritz $^{1}$, Alexander Schmidt ${ }^{1}$ and Christoph Handschin ${ }^{1, *}$

${ }^{1}$ Biozentrum, University of Basel, Basel, 4056, Switzerland.

*Co-corresponding author: j.perezschindler@unibas.ch; christoph.handschin@unibas.ch 


\section{SUMMARY}

Non-alcoholic fatty liver disease is continuum of disorders among which non-alcoholic steatohepatitis (NASH) is particularly associated with a negative prognosis. Hepatocyte lipotoxicity is one of the main pathogenic factors of liver fibrosis and NASH. However, the molecular mechanisms regulating this process are poorly understood. Here, we integrated transcriptomic and chromatin accessibility analyses from human liver and mouse hepatocytes to identify lipotoxicity-sensitive transcription factors. We found that the transcription factors MAFK and TCF4 were activated in liver from NASH patients and by mouse hepatocyte lipotoxicity. Genetic deletion of these transcription factors protected hepatocytes against saturated fatty acid oversupply. Notably, MAFK- and TCF4-regulated gene expression linked to lipotoxicity closely correlated with transcriptional patters in fibrosis progression in NASH patients. Collectively, our findings uncovered novel molecular insights into lipotoxicityinduced NASH, revealing the relevance and therapeutic potential of MAFK and TCF4 in human disease.

\section{INTRODUCTION}

Non-alcoholic fatty liver disease (NAFLD) is a spectrum of disorders that progresses from NAFL (hepatic steatosis) to non-alcoholic steatohepatitis (NASH), leading to cirrhosis and liver cancer (Friedman et al., 2018). NASH is characterized by chronic liver damage, inflammation and fibrosis, a stage that significantly increases morbidity and mortality (Friedman et al., 2018). Several genetic and environmental factors (e.g. a sedentary lifestyle) drive the development of NAFLD, among which the excessive supply of saturated fatty acids to the liver and, consequently, hepatocyte lipotoxicity is the main risk factor of NASH (Friedman et al., 2018; Suzuki and Diehl, 2017). Importantly, lipotoxicity is the leading cause of liver fibrosis that remains as the main predictor of liver-related death (Friedman et al., 2018; 
Suzuki and Diehl, 2017). However, therapies to target this process are scarce, as evidenced by the lack of approved medications for the treatment of NASH (Vuppalanchi et al., 2021).

Although the molecular underpinnings controlling hepatocyte lipotoxicity are elusive, key biological processes such as oxidative stress, endoplasmic reticulum (ER) stress and inflammation are known to play a central role (Friedman et al., 2018; Suzuki and Diehl, 2017). Interestingly, obesogenic diets extensively remodel the transcriptome, epigenome and chromatin accessibility landscape of mouse liver (Cazanave et al., 2017; Leung et al., 2014; Leung et al., 2016; Siersbaek et al., 2017). These studies suggest that disruption of transcriptional networks and chromatin function are fundamental to pathogenic progression. Such transcriptional networks are regulated by transcription factors that, upon activation, are recruited to DNA regulatory elements located in regions of accessible chromatin (Haberle and Stark, 2018). Despite the great therapeutic potential, the transcriptional mechanisms regulating lipotoxicity-induced NASH remain virtually unknown. Therefore, the main aim of this study was to identify lipotoxicity-sensitive transcription factors linked to the development of NASH. We achieved this aim by implementing a multi-omics approach in which human and mouse datasets were integrated to uncover conserved transcriptional mechanisms and potential therapeutic targets.

\section{RESULTS}

\section{NASH and lipotoxicity share a common molecular signature}

To discover conserved hepatocyte-specific mechanisms controlling NASH development, we implemented a multi-omics strategy in which we integrated analyses from human liver biopsies and mouse hepatocytes. Liver tissue was obtained from healthy control (CON) subjects and NASH patients suffering from obesity and diabetes (Figure 1A). In addition, lipotoxicity was investigated in FL83B mouse hepatocytes that were stimulated with bovine serum albumin 
bioRxiv preprint doi: https://doi.org/10.1101/2021.03.24 436772; this version posted March 24, 2021. The copyright holder for this preprint (which was not certified by peer review) is the author/funder, who has granted bioRxiv a license to display the preprint in perpetuity. It is made available under aCC-BY-NC-ND 4.0 International license.

(BSA) or BSA conjugated with the saturated fatty acid palmitate (PAL) for $24 \mathrm{~h}$. As expected, PAL induced a dose-dependent increase in hepatocyte cytotoxicity (Figure 1B). We found that while a low dose of PAL increases the accumulation of intracellular lipids, incubating cells with a higher dose had no significant effect (Figure 1C). Consistently, a lower capacity to incorporate saturated fatty acids into triglyceride has been demonstrate to mediate the cytotoxic effect of PAL overload (Listenberger et al., 2003). Notably, unless otherwise stated, all of the following experiments were carried out under high PAL concentration $(0.4 \mathrm{mM})$.

We initially assessed whether human liver from NASH patients and mouse hepatocyte lipotoxicity share a common transcriptional response. In humans, NASH was associated with the up- and down-regulation of 422 and 615 genes, respectively (Figure 1D). Compared to these chronic changes in human NASH, mouse hepatocytes exhibited a stronger acute transcriptional response as evidenced by a higher number of differentially expressed genes (DEG), comprising 1744 and 1396 up- and down-regulated genes, respectively (Figure 1E). Nevertheless, Gene Ontology (GO) analysis revealed several biological processes commonly regulated in both NASH and hepatocyte lipotoxicity. Consistent with the pathogenesis of $\mathrm{NASH}$, up-regulated genes were related to gluconeogenesis and oxidation-reduction process (Figure 1H), while down-regulated genes were linked to processes such as cell cycle progression and survival (Figure 1I). We next assessed the liver and hepatocyte proteome to complement the transcriptome analysis and improve the comparison between NASH and lipotoxicity. We found that proteome remodelling was also milder in liver from NASH patients (up-regulated: 92 and down-regulated: 191) compared to the effects of PAL stimulation in mouse hepatocytes (up-regulated: 830 and down-regulated: 552) (Figure 1F, 1G). A number of biological processes sensitive to both NASH and lipotoxicity were identified among upregulated proteins, including metabolic pathways also induced at the RNA level (Figure $1 \mathrm{~J}$ ). Moreover, our data demonstrate that down-regulated proteins were characterized by 
bioRxiv preprint doi: https://doi org/10.1101/2021.03.24 436772; this version posted March 24, 2021. The copyright holder for this preprint

dysregulation of common biological processes involved in oxidative stress, apoptosis and protein processing (Figure 1K). Importantly, common biological processes revealed by our analyses include key hallmarks of NASH (e.g. gluconeogenesis and ER stress), validating the cell culture model of hepatocyte lipotoxicity as a suitable system to discover and test potential therapeutic targets.

\section{NASH and lipotoxicity remodel the chromatin accessibility landscape}

We next performed chromatin accessibility analysis by assay for transposase-accessible chromatin by sequencing (ATAC-seq) to elucidate transcriptional mechanisms mediating lipotoxicity-induced NASH. The total number of ATAC-seq peaks (representing regions of accessible chromatin) was similar between liver from CON and NASH (Figure 2A). In contrast, mouse hepatocytes treated with PAL had a lower number of peaks compared to the BSA control condition (Figure 2B). Both human and mouse ATAC-seq data showed that the vast majority of peaks were located in introns and DNA regulatory elements such as promoters and enhancers, at distal intergenic regions (Figure 2A and 2B). Interestingly, our data revealed that the proportion of genomic regions (e.g. introns and promoters) harbouring accessible chromatin is to a large extent unaltered by NASH or lipotoxicity (Figure 2A and 2B). Globally, chromatin accessibility was slightly increased and strongly decreased in liver from NASH patients and in hepatocyte lipotoxicity, respectively (Figure 2C and 2D). Notably, in human livers, around $20 \%$ of the peaks were exclusively found in CON or NASH samples (Figure 2E and $2 \mathrm{G})$. These data demonstrate that although the total number of ATAC-seq peaks is similar between groups, there are specific regions on the chromatin in which accessibility is lost (CONspecific peaks) or gained (NASH-specific peaks) during NASH development. In the context of mouse hepatocyte lipotoxicity, we observed a higher proportion of BSA-specific (35\%) than PAL-specific (13\%) peaks (Figure $2 \mathrm{~F}$ and $2 \mathrm{H}$ ), which is consistent with the global decrease in chromatin accessibility induced by PAL. 
bioRxiv preprint doi: https://doi.org/10.1101/2021.03.24 436772; this version posted March 24,2021. The copyright holder for this preprint

To determine the link between changes in chromatin accessibility and gene expression, we identified down- and up-regulated genes associated with chromatin regions with decreased and increased accessibility, respectively. This analysis showed that repressed genes linked to CONspecific peaks were related to the regulation of cellular integrity, growth and vascularization, whereas up-regulated genes linked to NASH-specific peaks were mainly related to metabolic pathways (Figure 2I). Hepatocytes stimulated with PAL also exhibited a subset of downregulated genes linked to BSA-specific peaks that we found to be primarily associated with the regulation of cell cycle and survival (Figure 2J). Moreover, genes up-regulated by PAL and linked to PAL-specific peaks were involved in the positive regulation of the unfolded protein response (UPR) and the development of fibrosis (Figure 2J), thus supporting the role of hepatocyte lipotoxicity in NASH development. Our data therefore further support the strong transcriptional component in hepatocyte lipotoxicity-induced NASH.

\section{Lipotoxicity regulates a specific subset of transcription factors}

Our findings strongly suggest that transcription factors play a central regulatory role in hepatocyte lipotoxicity, a process that holds great therapeutic potential for the treatment of NASH (Vuppalanchi et al., 2021). We sought to identify such transcription factors via motif enrichment analysis of ATAC-seq peaks within each of the independent datasets generated from human liver and mouse hepatocytes. The overlap between these datasets uncovered 227 motifs representing 195 transcription factors commonly enriched both in human liver and mouse hepatocytes (Figure 3A). To refine the discovery of lipotoxicity-sensitive transcription factors, we used our transcriptomic data to perform transcription factor activity analysis via Integrated System for Motif Activity Response Analysis (ISMARA) (Balwierz et al., 2014). This approach identified 36 and 172 motifs representing 63 and 249 transcription factors predicted to have altered activity in NASH and lipotoxicity, respectively. By integrating these analyses, we found 35 transcription factors deregulated by NASH and lipotoxicity, among 
bioRxiv preprint doi: https://doi.org/10.1101/2021.03.24 436772; this version posted March 24,2021. The copyright holder for this preprint (which was not certified by peer review) is the author/funder, who has granted bioRxiv a license to display the preprint in perpetuity. It is made available under aCC-BY-NC-ND 4.0 International license.

which a subset of 13 also exhibited motif enrichment within regions of accessible chromatin (Figure 3A). Clustering analysis based on ISMARA-predicted activity showed a small fraction of transcription factors in which NASH and PAL induced the same effect (Figure 3B). Assessment of changes in the RNA levels of these transcription factors implies that, generally, the predicted activity does not directly relate to gene expression patterns (Figure 3B). Transcription factors sharing the same response might reflect redundant regulators due to similarities in their corresponding binding motifs on the DNA. We thus performed clustering analysis based on the consensus sequence of such motifs. Although we were able to define 5 distinct clusters, they did not match clustering patterns based on inferred transcription factor activity (Figure S1A). This suggests that most of these transcription factors regulate unique transcriptional networks related to hepatocyte lipotoxicity.

Subsequently, we selected a subset of transcription factor candidates that exhibit equal changes in activity both in NASH and lipotoxicity. We then either overexpressed (OE) repressed transcription factors (EGR2, KLF4, NFYA, NFYB, SP3 and TFEB) or knocked out (KO) those that were activated (MAFK and TCF4) in mouse hepatocytes (Figure S1B). This strategy allowed us to determine whether $\mathrm{OE}$ or $\mathrm{KO}$ of candidate transcription factors would confer protection against PAL-induced lipotoxicity. Although EGR2, NFYA and NFYB OE decreased the cytotoxic response to a lower dose of PAL, none of the candidates protected cells against the higher dose when OE (Figure 3C). In contrast, we found that genetic deletion of either MAFK or TCF4 was sufficient to lower the cytotoxic response to the high dose of PAL in mouse hepatocytes (Figure 3C). When EGR2, NFYA and NFYB were OE, we observed a slight increased content of intracellular lipids under low PAL conditions (Figure S1C), suggesting a possible link to the protective effects against lipotoxicity. KO of MAFK or TCF4 on the other hand had no effect on intracellular lipid storage under the experimental conditions in which they ameliorate lipotoxicity (Figure S1C). To further assess the biomedical relevance 
bioRxiv preprint doi: https://doi org/10.1101/2021.03.24 436772; this version posted March 24, 2021. The copyright holder for this preprint (which was not certified by peer review) is the author/funder, who has granted bioRxiv a license to display the preprint in perpetuity. It is made available under aCC-BY-NC-ND 4.0 International license.

of MAFK and TCF4, we carried out ISMARA analysis with a different RNA-seq dataset (GEO accession: GSE126848) from a large cohort of human liver samples, comprising a mild (NAFL) and advance (NASH) stage of NAFLD (Suppli et al., 2019). Supporting our results, the predicted activity of MAFK and TCF4 was significantly increased in human liver from both NAFL and NASH patients (Figure 3D). This independent validation of our findings further demonstrates that MAFK and TCF4 are lipotoxicity-sensitive transcription factors mediating a pathological effect that is conserved between human and mouse.

\section{MAFK and TCF4 regulate distinct transcriptional networks linked to NASH}

In order to define the molecular mechanisms by which MAFK and TCF4 regulate lipotoxicity, we performed transcriptome analysis of $\mathrm{CON}$ and $\mathrm{KO}$ cells in the absence (BSA) or presence of PAL at high concentration $(0.4 \mathrm{mM})$. TCF4 KO had a mild effect under basal condition, inducing the up- and down-regulation of 106 and 27 genes, respectively (Figure S2A). The total number of DEG induced by PAL was slightly higher in TCF4-KO cells, with about 26\% of DEG being regulated in a TCF4-sensitive manner (Figure 4A, S2B and S2C). Moreover, direct comparison of cells stimulated with PAL revealed 80 and 97 gene showing a higher and lower expression in TCF4-KO cells, respectively (Figure 4B), representing commonly regulated genes also sensitive to TCF4 deletion. We grouped all TCF4-sensitive genes and performed clustering analysis to identify subsets of genes regulated by PAL and showing an evident dependency on TCF4. Among the 4 main clusters identified by this analysis, cluster 1 was mainly characterized by genes in which the up-regulation induced by PAL was prevented in TCF4-KO cells (Figure 4C). GO analysis of cluster 1 uncovered translation as a key biological process regulated by these genes (Figure 4D). Considering the importance of liver fibrosis in NASH prognosis, we next defined the association between TCF4-sensitive genes and human NASH at different stages of fibrosis. We leveraged RNA-seq data (GEO accession: GSE135251) from a large human study comprising liver samples from NASH patients with 
bioRxiv preprint doi: https://doi org/10.1101/2021.03.24 436772; this version posted March 24, 2021. The copyright holder for this preprint (which was not certified by peer review) is the author/funder, who has granted bioRxiv a license to display the preprint in perpetuity. It is made available under aCC-BY-NC-ND 4.0 International license.

mild (F2), advanced (F3) and severe (F4) fibrosis (Govaere et al., 2020). Among common DEG in humans NASH F4 and mouse hepatocyte lipotoxicity, the up- and down-regulation of 17 and 3 was controlled by TCF4, respectively (Figure 4E). Interestingly, the expression of TCF4sensitive genes appears to be regulated in a fibrosis-dependent manner, where a higher degree of fibrosis is associated with a stronger effect in human liver (Figure 4F). While CON hepatocytes stimulated with PAL recapitulated the expression patterns observed in liver from NASH F4 patients, genetic deletion of TCF4 was able to counteract such a response (Figure $4 \mathrm{~F})$.

We next carried out an analogous characterisation of MAFK-KO hepatocytes in the context of lipotoxicity. KO of MAFK under basal conditions resulted in the up- and down-regulation of 37 and 30 genes, respectively (Figure S2D). Transcriptome remodelling of PAL-stimulated cells was slightly higher in CON and, similarly to TCF4-KO cells, about $20 \%$ of DEG were regulated in a MAFK-sensitive manner (Figure S2E, S2F and S3A). We additionally found 21 and 17 up- and down-regulated genes, respectively, in MAFK-KO cells when compared to CON, both following PAL stimulation (Figure S3B). Hierarchical clustering of MAFKsensitive genes uncovered 4 prominent groups, among which cluster 3 showed a robust difference between CON and MAFK-KO cells in the context of lipotoxicity (Figure S3C). This cluster only contained 21 genes characterized by a down-regulation in MAFK-KO cells under basal condition, with a small fraction up-regulated exclusively in CON cells in response to PAL (Figure S3D). When compared to human NASH F4, we identified 13 and 2 up- and downregulated genes, respectively, regulated in a MAFK-depended manner (Figure S3E). MAFKsensitive gene regulation was also closely correlated to fibrosis in human liver, with genetic deletion of this transcription factor effectively attenuating the effects of lipotoxicity (Figure S3F). Notably, besides supporting the relevance of MAFK and TCF4, transcriptomic analysis 
of KO cells revealed a wide range of novel genes and pathways involved in the development of hepatocyte lipotoxicity.

\section{DISCUSSION}

Despite the alarming increase in the worldwide prevalence of NASH, to date there are no approved medications for the treatment of this devastating disease (Vuppalanchi et al., 2021). NASH development is mediated by a wide range of factors, among which hepatocyte lipotoxicity is the main predictor of fibrosis and, consequently, liver-related mortality (Vuppalanchi et al., 2021). The overload of intracellular lipids in hepatocytes due to an oversupply of saturated fatty acids and/or enhanced de novo lipogenesis (e.g. high fructose consumption) induces the acute activation of pathway promoting cellular injury and death such as the UPR, oxidative stress and pro-inflammatory signals (Friedman et al., 2018). However, the lack of knowledge regarding transcriptional mechanism in lipotoxicity has limited the identification of therapeutic targets regulating this fundamental pathogenic process in NASH. Moreover, an important obstacle in the discovery and validation of new therapeutic targets for NASH is that the current preclinical mouse models poorly recapitulate disease development in humans. In fact, most mouse models fail to concurrently exhibit all key metabolic and histological hallmarks of human $\mathrm{NASH}$, such as insulin resistance, steatosis, fibrosis, inflammation and hepatocyte ballooning (Im et al., 2020; Santhekadur et al., 2018). To circumvent some of these shortcomings, the analysis of acute changes triggered by lipotoxicity in hepatocytes was closely linked to the chronic transcriptional modulation observed in human NASH in situ. Thereby, a very stringent stratification of transcriptional programs and regulators was integrated. While this stringency might result in false negative calling of candidates due to the focus on hepatocytes and acute effects of PAL, this combined approach will reveal transcription factor featuring a regulation and function with a robust relevance for the human disease. Importantly, the integration of transcriptome and chromatin accessibility 
bioRxiv preprint doi: https://doi.org/10.1101/2021.03.24 436772; this version posted March 24,2021. The copyright holder for this preprint (which was not certified by peer review) is the author/funder, who has granted bioRxiv a license to display the preprint in perpetuity. It is made available under aCC-BY-NC-ND 4.0 International license.

analyses allowed us to discover lipotoxicity-sensitive transcription factors and their downstream transcriptional networks in hepatocytes. In addition, the rigorous cross-validation of our findings with independent human datasets then substantially strengthened their biomedical relevance, with MAFK and TCF4 emerging as novel targets with high therapeutic potential in NASH.

Liver metabolism and function is regulated by a variety of transcriptional factors, some of which have been implicated in the development of NAFLD (Wang et al., 2015; Xiao et al., 2020). The pathogenic relevance of transcription factors is supported by several studies demonstrating that NAFLD is associated with altered chromatin function and, thus, gene expression (Cazanave et al., 2017; Leung et al., 2014; Leung et al., 2016; Siersbaek et al., 2017). Our data demonstrate that, in hepatocytes, both TCF4 and MAFK are highly sensitive to saturated fatty acids supply. To our knowledge, none of these transcription factors have been implicated in the development of NASH. Of particular interest, we found that, in the context of lipotoxicity, TCF4 regulated the expression of a subset of genes enhancing protein synthesis. An abnormal increase in protein synthesis can overload the ER and thereby activate the UPR (Appenzeller-Herzog and Hall, 2012; Hetz et al., 2020). Interestingly, hepatocyte lipotoxicity has been associated with the activation of the mammalian target of rapamycin complex 1 (mTORC1) (Ao et al., 2020; Chen et al., 2020), a master regulator of protein synthesis (Liu and Sabatini, 2020). In line with the link between enhanced protein synthesis and lipotoxicity, pharmacological inhibition of mTORC1 protected hepatocytes against the toxic effects of palmitate (Ao et al., 2020; Chen et al., 2020). Moreover, genetic activation of mTORC1 and 2 in mouse liver strongly induces fibrosis and NASH (Guri et al., 2017). It is thus possible that, besides the acute activation of mTORC1, TCF4 stimulation by lipotoxicity further enhances the UPR by increasing the expression of genes controlling protein synthesis. Unlike TCF4, MAFK-sensitive genes did not reveal a particular annotated deregulated pathway in the context 
bioRxiv preprint doi: https://doi org/10.1101/2021.03.24 436772; this version posted March 24, 2021. The copyright holder for this preprint (which was not certified by peer review) is the author/funder, who has granted bioRxiv a license to display the preprint in perpetuity. It is made available under aCC-BY-NC-ND 4.0 International license.

of lipotoxicity. Nevertheless, among genes up-regulated by PAL showing a blunted response in MAFK-KO cells, we found crucial regulators of processes determining cellular health such as autophagy and apoptosis (e.g. Pink1, Atg10, Atg101, Foxo3 and Aatk). Remarkably, beyond known pathways linked to NASH, our global unbiased analyses revealed a wide collection MAFK- and TCF4-sensitive gene representing novel therapeutic targets to fight liver lipotoxicity.

An important strategy of our study was to determine candidate transcription factors and downstream target genes exhibiting a conserved response to NASH/lipotoxicity between human liver and mouse hepatocytes. Candidates showing such level of conservation are expected to play a central role in disease development, thus holding great therapeutic potential. In line with this idea, we found several genes with a known regulatory role in liver fibrosis (Sema7a, Cdh11) (De Minicis et al., 2013; Pedroza et al., 2019; Ruan et al., 2019) and NAFLD development (Adam28, Lgals3, Cxcl10) (Herat et al., 2017; Iacobini et al., 2011; Jeftic et al., 2015; Zhang et al., 2014) among MAFK- and TCF4-sensitive genes commonly regulated in human NASH. Notably, while the TCF4-sensitive gene Cxcl10 has been proposed as a NASH biomarker (Zhang et al., 2014), the Lgals3 (also known as Galectin 3) inhibitor Belapectin is a promising medication for NASH currently under investigation and development for phase 3 clinical trials (Al Attar et al., 2021; Vuppalanchi et al., 2021). These studies strongly support our experimental approach as an efficient strategy to discover promising therapeutic targets for NASH in humans.

In conclusion, our findings have uncovered a new layer of complexity in lipotoxicity-induced NASH, where transcriptional networks sensitive to saturated fatty acids promote cell death and fibrosis. By performing thorough comparisons to human datasets and experimental validation, the transcription factors MAFK and TCF4 emerged as central regulators of hepatocyte lipotoxicity and link the MAFK/TCF4 transcriptional networks to the development of liver 
fibrosis. These transcription factors therefore are highly attractive targets to ameliorate NASH and liver-related death. Furthermore, our data provide a rich resource to generate new hypotheses for future studies aiming at tackling liver lipotoxicity, the central pathogenic factor of NASH.

\section{METHODS}

\section{Human samples}

Fresh frozen and pulverized human liver samples were purchased from Sekisui XenoTech. Samples were obtained from normal (lot \#: H1281, H1296, H1310 and H1336) and nonalcoholic steatohepatitis (lot \#: H0847, H0958, H1027 and H1060) donors with appropriate consent and ethical approval obtained by Sekisui XenoTech.

\section{Cell culture and palmitate treatment}

FL83B mouse hepatocytes (ATCC ${ }^{\circledR}, \#$ CRL-2390 ${ }^{\mathrm{TM}}$ ) were grown in F-12K medium (Thermo Fisher Scientific, \#21127030) supplemented with 10\% fetal bovine serum (growth medium). Cells were maintained at $37^{\circ} \mathrm{C}, 95 \% \mathrm{O}_{2}$ and $5 \% \mathrm{CO}_{2}$.

A stock solution of $25 \mathrm{mM}$ sodium palmitate (PAL) (Sigma-Aldrich, \#P9767) was prepared in serum-free F-12K media containing $1 \%$ fatty acid free bovine serum albumin (BSA) (SigmaAldrich, \#A8806), which was incubated at $70^{\circ} \mathrm{C}$ for 30 min shaking at $1000 \mathrm{rpm}$ before use. Next, cells were incubated in 0, 200 and $400 \mu \mathrm{M}$ PAL diluted in growth medium for $24 \mathrm{~h}$. One percent BSA diluted in growth medium was used as control.

\section{Cytotoxicity assay}

Cytotoxicity was measured with CellTox ${ }^{\mathrm{TM}}$ Green Cytotoxicity Assay (Promega, \#G8742), according to the manufacturer's instructions.

\section{Intracellular lipid staining}


Cells were incubated with a staining solution containing $1 \mu \mathrm{g} / \mathrm{ml}$ of Hoechst 33342 (Thermo Fisher Scientific, \#H3570) and $500 \mathrm{ng} / \mathrm{ml}$ of Nile Red (Sigma-Aldrich, \#N3013) in phosphate buffered saline (PBS) for 20 min covered from light at $37{ }^{\circ} \mathrm{C}$ and $5 \% \mathrm{CO}_{2}$. Next, staining solution was removed and cells were washed twice with PBS. Fresh PBS was added before measuring Nile Red (excitation/emission $=488 / 550 \mathrm{~nm}$ ) and Hoechst 33342 (excitation/emission $=350 / 461 \mathrm{~nm}$ ) fluorescence with a microplate reader. Nile Red signal (lipids) was normalized to Hoechst 33342 signal (DNA) to account for differences in cell number.

\section{Transient transfections}

Transfection of FL83B cells was performed using Opti-MEM ${ }^{\mathrm{TM}}$ (Thermo Fisher Scientific, \#31985070) and polyethylenimine (Polysciences, \# 23966). Plasmids and polyethylenimine were diluted in Opti-MEM ${ }^{\mathrm{TM}}$, following which they were mixed in a 1:3 ratio of ug DNA:ug polyethylenimine and incubated for $20 \mathrm{~min}$ at room temperature before adding to the cells. Cells were transfected $24 \mathrm{~h}$ after seeding with 0.1 or $1 \mu \mathrm{g}$ per well of a 96 and 12 well plate, respectively, of pcDNA 3.1, pAd-Klf4 (Addgene, \# 19770, gift from Konrad Hochedlinger), pFLAG/HA/mSp3 (VectorBuilder, \#VB200204-1104aty), pFLAG/HA/mTfeb (VectorBuilder, \#VB200204-1105cfn), pFLAG/HA/mEgr2 (VectorBuilder, \#VB2002041106upe), pFLAG/HA/mNfya (VectorBuilder, \#VB200204-1107xne) or pFLAG/HA/mNfyb (VectorBuilder, \#VB200204-1108efg) for a total of $48 \mathrm{~h}$. Palmitate treatment was started $24 \mathrm{~h}$ after transfection.

\section{Generation of knockout cells}

Gene knockout (KO) was achieved by using the CRISPR-Cas9 system. To KO the mouse Klf4 gene, FL83B cells were transfected as described above with the following plasmids: p-hCas9mTcf4-gRNA\#32572-mTcf4-gRNA\#30405 (VectorBuilder, \#VB200206-1079fgw) and p- 
hCas9-Scramble-gRNA1-Scramble-gRNA2 (VectorBuilder, \#VB200206-1080bvj) as nontargeting control. Deletion of the mouse Mafk gene was performed using the Edit-R gene editing system (Horizon Discovery). First, stable Cas9 expression was induced by transducing FL83B cells with lentiCas9-Blast (Addgene, \#52962-LV, gift from Feng Zhang) lentivirus at a multiplicity of infection (MOI) of 0.5 in growth medium containing $10 \mu \mathrm{g} / \mathrm{ml}$ of polybrene (Sigma-Aldrich, \#107689). Next, cells were selected with $6 \mu \mathrm{g} / \mathrm{ml}$ of blasticidin (Thermo Fisher Scientific, \#A1113903) and a monoclonal cell line was obtained via limiting dilution. Cas9 expressing FL83B cells were subsequently transduced with Mafk (\#VSGM10144246720110) or non-targeting control (\#VSGC10215) lentiviral sgRNA at an MOI of 0.5 in growth medium containing $10 \mu \mathrm{g} / \mathrm{ml}$ of polybrene. Seventy two hours after Tcf4 or Mafk targeting, cells were selected with $1 \mu \mathrm{g} / \mathrm{ml}$ of puromycin (Thermo Fisher Scientific, \#A1113803) to generate a polyclonal population of KO cells for downstream experiments.

\section{RNA purification and quantitative PCR (qPCR)}

Liver tissue and cells were lysed in $1 \mathrm{ml}$ of TRI Reagent (Sigma \#T9424) and incubated for 5 min at room temperature. Aqueous phase was obtained with chloroform following the manufacturer's instructions, following which RNA was purified and reverse transcribed using Direct-zol $^{\mathrm{TM}}$ RNA MiniPrep (Zymo Research, \#R2050) and iScript ${ }^{\mathrm{TM}}$ cDNA Synthesis Kit (Bio-Rad, \#1708891), respectively. Relative changes in mRNA content was quantified by qPCR on a StepOnePlus system (Applied Biosystems) using Fast SYBR ${ }^{\text {TM }}$ Green Master Mix (Thermo Fisher Scientific, \#4385612). The $\Delta \Delta C$ T method was used for analysis, with TATA binding protein $(T b p)$ as endogenous control.

\section{RNA sequencing (RNA-seq)}

Libraries from human liver tissue and wild type FL83B cells were prepared with TruSeq Stranded Total RNA Library Prep Gold (Illumina, \#20020599), single-read sequencing was 
performed using the NextSeq 500 (Illumina). Libraries from knockout FL83B cells and their corresponding non-targeting controls were prepared with TruSeq Stranded mRNA Library Kit (Illumina, \#20020595), pair-end sequencing was performed using the NovaSeq 6000 (Illumina). All data was analysed on the Galaxy platform (https://usegalaxy.eu/). Reads were trimmed with Trim Galore! (Galaxy version 0.4.3.1) and quality was assessed using FastQC (Galaxy version $0.72+$ galaxy1). Reads were aligned to the hg38 or mm10 version of the human and mouse genome, respectively, using STAR (Galaxy version 2.7.7a). Strand specificity and read counting was performed with Infer Experiment (Galaxy version 2.6.4.1) and featureCounts (Galaxy version 2.0.1), respectively. Next, we used DESeq2 (Galaxy version 2.11.40.6+galaxy1) for differential expression analysis (fold change $\geq 1.5$, p-value $<0.05$ for human liver tissue and q-value $<0.05$ for FL83B cells) and the resulting data was annotated with Annotate DESeq2/DEXSeq output tables (Galaxy version 1.1.0). Overlap between different datasets was determined with Venny (version 2.1, https://bioinfogp.cnb.csic.es/tools/venny/) and volcano plots generated with Volcano Plot (Galaxy Version 0.0.3). Gene Ontology (GO) analysis was performed with DAVID 6.8 (https://david.ncifcrf.gov/), with significance defined as p-value $<0.05$. Transcription factors activity analysis was achieved with ISMARA (https://ismara.unibas.ch/mara/), where z-value $\geq 1.5$ was consider as significant. We used DESeq2 normalized counts to generate heat maps and for hierarchical clustering using Morpheus (https://clue.io/morpheus).

\section{Mass spectrometry analysis of whole cell proteome}

Fifteen milligrams of powdered human liver tissue were lysed in $200 \mathrm{uL}$ of lysis buffer $(8 \mathrm{M}$ Urea, $50 \mathrm{mM}$ Tris- $\mathrm{HCl} \mathrm{pH} 7.5,150 \mathrm{mM} \mathrm{NaCl}$ and $1 \mathrm{X}$ Halt ${ }^{\mathrm{TM}}$ Protease Inhibitor Cocktail (Thermo Fisher Scientific, \#87786)), vortexed at max speed for $15 \mathrm{sec}$ and incubated for 30 min at $4{ }^{\circ} \mathrm{C}$ with shaking at $14000 \mathrm{rpm}$. Next, samples were sonicated (amplitude $100 \%$, Cycle 0.5) four times for $30 \mathrm{sec}$ in a vial tweeter sonicator with 2 min pause on ice between cycles. 
Samples were then centrifuged for $13000 \mathrm{~g}$ for $10 \mathrm{~min}$ at $4^{\circ} \mathrm{C}$, supernatant was transferred to a new tube and the pellet was homogenized in $50 \mu \mathrm{l}$ of lysis buffer. Following an additional centrifugation at $13000 \mathrm{~g}$ for $10 \mathrm{~min}$ at $4^{\circ} \mathrm{C}$, supernatant was combined with the previous supernatant. Samples were centrifuged a final time at $13000 \mathrm{~g}$ for $10 \mathrm{~min}$ at $4^{\circ} \mathrm{C}$ and supernatant was used for mass spectrometry. On the other hand, mouse hepatocytes were seeded in 6 well plates and harvested in $80 \mu \mathrm{l}$ of lysis buffer per well (1\% sodium deoxycholate (SDC), $0.1 \mathrm{M}$ TRIS, $10 \mathrm{mM}$ TCEP, $\mathrm{pH}=8.5$ ), following lysis with 10 cycles of sonication (Bioruptor, Diagnode). All samples were reduced for $10 \mathrm{~min}$ at $95^{\circ} \mathrm{C}$ and alkylated at $15 \mathrm{mM}$ chloroacetamide for $30 \mathrm{~min}$ at $37^{\circ} \mathrm{C}$. Proteins were digested by incubation with sequencinggrade modified trypsin $\left(1 / 50 \mathrm{w} / \mathrm{w}\right.$; Promega,V5113) for $12 \mathrm{~h}$ at $37^{\circ} \mathrm{C}$. Tryptic digests were acidified $(\mathrm{pH}<3)$ using TFA and cleaned up using iST cartridges (PreOmics, P.O.00027) according to the manufacturer's instructions. Samples were dried under vacuum and stored at $-20{ }^{\circ} \mathrm{C}$.

Sample aliquots comprising $25 \mu \mathrm{g}$ of peptides were labelled with isobaric tandem mass tags (TMT 10-plex, Thermo Fisher Scientific, \#90110) as described previously (Ahrne et al., 2016). Shortly, peptides were re-suspended in $20 \mu$ labelling buffer ( $2 \mathrm{M}$ urea, $0.2 \mathrm{M}$ HEPES, pH 8.3) and $5 \mu \mathrm{L}$ of each TMT reagent were added to the individual peptide samples followed by a $1 \mathrm{~h}$ incubation at $25^{\circ} \mathrm{C}$, shaking at $500 \mathrm{rpm}$. To quench the labelling reaction, $1.5 \mu \mathrm{L}$ aqueous 1.5 M hydroxylamine solution was added and samples were incubated for another $10 \mathrm{~min}$ at $25^{\circ} \mathrm{C}$ shaking at $500 \mathrm{rpm}$ followed by pooling of all samples. The $\mathrm{pH}$ of the sample pool was increased to 11.9 by adding $1 \mathrm{M}$ phosphate buffer $(\mathrm{pH} 12)$ and incubated for $20 \mathrm{~min}$ at $25^{\circ} \mathrm{C}$ shaking at $500 \mathrm{rpm}$ to remove TMT labels linked to peptide hydroxyl groups. Subsequently, the reaction was stopped by adding $2 \mathrm{M}$ hydrochloric acid until a $\mathrm{pH}<2$ was reached. Finally, peptide samples were further acidified using $5 \%$ TFA, desalted using Sep-Pak Vac 1cc (50 
mg) C18 cartridges (Waters, \#WAT054960) according to the manufacturer's instructions and dried under vacuum.

TMT-labelled peptides were fractionated by high-pH reversed phase separation using a XBridge Peptide BEH C18 column (3,5 $\mu \mathrm{m}, 130 \AA$ Å, 1 mm x 150 mm; Waters, \#186003562) on an Agilent 1260 Infinity HPLC system. Peptides were loaded on column in buffer A (20 $\mathrm{mM}$ ammonium formate in water, $\mathrm{pH} 10$ ) and eluted using a two-step linear gradient from $2 \%$ to $10 \%$ in $5 \mathrm{~min}$ and then to $50 \%$ buffer $\mathrm{B}$ (20 mM ammonium formate in $90 \%$ acetonitrile, $\mathrm{pH}$ 10) over $55 \mathrm{~min}$ at a flow rate of $42 \mu \mathrm{l} / \mathrm{min}$. Elution of peptides was monitored with a UV detector $(215 \mathrm{~nm}, 254 \mathrm{~nm})$ and a total of 36 fractions were collected, pooled into 12 fractions using a post-concatenation strategy as previously described (Wang et al., 2011) and dried under vacuum.

Dried peptides were re-suspended in $0.1 \%$ aqueous formic acid and subjected to LC-MS/MS analysis using a Q Exactive HF Mass Spectrometer fitted with an EASY-nLC 1000 (Thermo Fisher Scientific) and a custom-made column heater set to $60^{\circ} \mathrm{C}$. Peptides were resolved using a RP-HPLC column $(75 \mu \mathrm{m} \times 30 \mathrm{~cm})$ packed in-house with C18 resin (ReproSil-Pur C18-AQ, $1.9 \mu \mathrm{m}$ resin; Dr. Maisch, r119.aq.) at a flow rate of $0.2 \mu \mathrm{Lmin}-1$. The following gradient was used for separation of murine peptides: from $5 \% \mathrm{~B}$ to $15 \% \mathrm{~B}$ over $10 \mathrm{~min}$ to $30 \% \mathrm{~B}$ over 60 min to $45 \%$ B over 20 min to $95 \%$ B over 2 min followed by 18 min at $95 \%$ B, whereas the following gradient was used for separation of human peptides: from 5\% B to $15 \%$ B over 14 min to $30 \%$ B over 80 min to $45 \%$ B over $26 \min$ to $95 \%$ B over 2 min followed by 18 min at $95 \%$ B. Buffer A was $0.1 \%$ formic acid in water and buffer B was $80 \%$ acetonitrile, $0.1 \%$ formic acid in water.

The mass spectrometer was operated in DDA mode with a total cycle time of approximately 1 s. Each MS1 scan was followed by high-collision-dissociation (HCD) of the 10 most abundant precursor ions with dynamic exclusion set to $30 \mathrm{~s}$. For MS1, 3e6 ions were accumulated in the 
bioRxiv preprint doi: $h$ ttps://doi org/10.1101/2021.03.24.436772; this version posted March 24, 2021. The copyright holder for this preprint (which was not certified by peer review) is the author/funder, who has granted bioRxiv a license to display the preprint in perpetuity. It is made available under aCC-BY-NC-ND 4.0 International license.

Orbitrap over a maximum time of $100 \mathrm{~ms}$ and scanned at a resolution of 120,000 FWHM (at $200 \mathrm{~m} / \mathrm{z}$ ). MS2 scans were acquired at a target setting of $1 \mathrm{e} 5$ ions, maximum accumulation time of $100 \mathrm{~ms}$ and a resolution of 30,000 FWHM (at $200 \mathrm{~m} / \mathrm{z}$ ). Singly charged ions and ions with unassigned charge state were excluded from triggering MS2 events. The normalized collision energy was set to $35 \%$, the mass isolation window was set to $1.1 \mathrm{~m} / \mathrm{z}$ and one microscan was acquired for each spectrum.

The acquired raw-files were converted to the mascot generic file (mgf) format using the msconvert tool (part of ProteoWizard, version 3.0.4624 (2013-6-3)) and searched using MASCOT either against a murine database (consisting of 49434 forward and reverse protein sequences downloaded from Uniprot on 20141124) or a human database (consisting of 40832 forward and reverse protein sequences downloaded from Uniprot on 20181213) and 390 commonly observed contaminants. The precursor ion tolerance was set to $10 \mathrm{ppm}$ and fragment ion tolerance was set to $0.02 \mathrm{Da}$. The search criteria were set as follows: full tryptic specificity was required (cleavage after lysine or arginine residues unless followed by proline), 3 missed cleavages were allowed, carbamidomethylation (C) and TMT6plex (K and peptide N-terminus) were set as fixed modification and oxidation $(\mathrm{M})$ as a variable modification. Next, the database search results were imported into the Scaffold Q+ software (version 4.3.2, Proteome Software Inc.) and the protein false discovery rate was set to $1 \%$ based on the number of decoy hits. Proteins that contained similar peptides and could not be differentiated based on MS/MS analysis alone were grouped to satisfy the principles of parsimony. Proteins sharing significant peptide evidence were grouped into clusters. Acquired reporter ion intensities in the experiments were employed for automated quantification and statistical analysis using a modified version of our in-house developed SafeQuant R script v2.3 (Ahrne et al., 2016). This analysis included adjustment of reporter ion intensities, global data normalization by equalizing the total reporter ion intensity across all channels, summation of reporter ion intensities per 
protein and channel, calculation of protein abundance ratios and testing for differential abundance using empirical Bayes moderated t-statistics. The calculated p-values were corrected for multiple testing using the Benjamini-Hochberg method, with significance defined as fold change $\geq 1.3$, $\mathrm{p}$-value $<0.05$ for human liver tissue and q-value $<0.05$ for FL83B cells. Volcano plots were generated on the Galaxy platform (https://usegalaxy.eu/) with Volcano Plot (Galaxy Version 0.0.3). GO analysis was performed with DAVID 6.8 (https://david.ncifcrf.gov/), with significance defined as p-value $<0.05$. Overlap between different datasets was determined with Venny (version 2.1, https://bioinfogp.cnb.csic.es/tools/venny/).

\section{Assay for transposase-accessible chromatin by sequencing (ATAC-seq)}

Nuclei from human liver $(15 \mathrm{mg})$ were isolated using with the Nuclei EZ Prep kit (SigmaAldrich, \#NUC101) and a glass dounce homogenizer (25 strokes with lose pastel and 25 strokes with tight pastel in ice-cold Nuclei EZ Lysis Buffer). Nuclei were filter through a $40 \mu \mathrm{m}$ cell strainer and counted before ATAC-seq. On the other hand, F183B mouse hepatocytes were seeded in $60 \mathrm{~mm}$ plates $24 \mathrm{~h}$ before BSA or PAL treatment. Nuclei from human liver and mouse hepatocytes were then used for ATAC-seq and library preparation as previously described (Corces et al., 2017). Pair-end sequencing of the libraries was performed using the Illumina NextSeq 500. All data was analysed on the Galaxy platform (https://usegalaxy.eu/). Reads were trimmed with Cutadapt (Galaxy version: 1.16.5) and quality was assessed using FastQC (Galaxy version $0.72+$ galaxy1). Reads were aligned to the hg38 or mm10 version of the human and mouse genome, respectively, using Bowtie2 (Galaxy version 2.3.4.3+galaxy0). Low quality reads (phred < 30) were filtered out with Filter (Galaxy Version 2.4.1) and duplicated reads were removed with MarkDuplicates (Galaxy version 2.18.2.2). Next, we used Genrich (Galaxy version 0.5+galaxy2) for peak calling (q-value $<0.05$ ), while ChIPseeker (Galaxy version, \#1.18.0+galaxy1) was used to annotate peaks. The overlap between different 
ATAC-seq datasets was performed with bedtools Intersect intervals (Galaxy version 2.29.0). Heat map and density plots of ATAC-seq peaks were created with plotHeatmap (Galaxy version 3.0.2.0) and plotProfile (Galaxy version 3.1.2.0.0), respectively. Data was visualized on the Integrated Genome Browser-9.1.4 (Freese et al., 2016) to generate representative genome browser figures, for which BAM files were merged with Merge BAM Files (Galaxy version 1.2.0) and then normalized using bamCoverage (Galaxy version 3.0.2.0). CentriMo (version 5.1.0, https://meme-suite.org/meme/doc/centrimo.html) was used to perform motif enrichment analysis (E-value threshold < 0.05) (Bailey and Machanick, 2012). Finally, clustering of transcription factor motifs based in their consensus sequence was performed using STAMP (http://www.benoslab.pitt.edu/stamp/) (Mahony and Benos, 2007).

\section{Statistics}

All qPCR, cytotoxicity and intracellular lipid assays were performed at least three independent times each in triplicate. Number of replicates per experiment is indicated in the figure legend when appropriate. Values are expressed as mean \pm SD. Statistical significance was determined with unpaired two-tailed t-tests, with significance considered with a $\mathrm{p}<0.05$.

RNA-seq, ATAC-seq and mass spectrometry experiments were performed once with three to four biological replicates as indicated in figure legends. Statistical analysis of these experiments is described above in their corresponding sections.

\section{Data availability}

RNA-seq and ATAC-seq data will be deposited to the Gene Expression Omnibus. Whole proteome analysis will be deposited to PRIDE. All other data are available from the corresponding authors upon reasonable request. 


\section{ACKNOWLEDGEMENTS}

We thank Christian Beisel (Genomics Facility Basel, ETH Zürich) and Philippe Demougin (Life Sciences Training Facility, Biozentrum, University of Basel) for technical help. We thank Eva Dazert for advice about processing of human liver samples (Biozentrum, University of Basel). This work was supported by the Novartis Foundation for Medical-Biological Research (J.P.S.), Research Fund of the University of Basel (J.P.S.), the Swiss National Science Foundation, the European Research Council (ERC) Consolidator grant 616830MUSCLE_NET, Swiss Cancer Research grant KFS-3733-08-2015, the Swiss Society for Research on Muscle Diseases (SSEM), SystemsX.ch, the Novartis Stiftung für MedizinischBiologische Forschung and the University of Basel (C.H.). C.H.O. is supported by a research grant from the Danish Diabetes Academy, which is funded by the Novo Nordisk Foundation (Grant no. NNF17SA0031406).

\section{AUTHOR CONTRIBUTIONS}

JPS and $\mathrm{CH}$ conceived, designed and supervised the study. JPS, EVF, BKC, DR and AS performed experiments. JPS, DR, AS and $\mathrm{CH}$ performed data analysis and interpretation. JPS and $\mathrm{CH}$ wrote the manuscript. All authors reviewed the manuscript.

\section{DECLARATION OF INTERESTS}

The authors declare no competing interests 


\section{FIGURES}

\section{A}

\begin{tabular}{|l|c|c|}
\cline { 2 - 3 } \multicolumn{1}{c|}{} & \multicolumn{2}{c|}{ Human subjects } \\
\cline { 2 - 3 } \multicolumn{1}{c|}{} & CON $(\mathbf{n = 4})$ & NASH $(\mathbf{n = 4})$ \\
\hline Age (years) & $48.5 \pm 15.9$ & $53.5 \pm 7.2$ \\
\hline Gender & 2 male/2 female & 2 male/2 female \\
\hline BMI (kg/m2) & $22.1 \pm 1.4$ & $41.4 \pm 3.4$ \\
\hline Diabetes & No & Yes \\
\hline
\end{tabular}
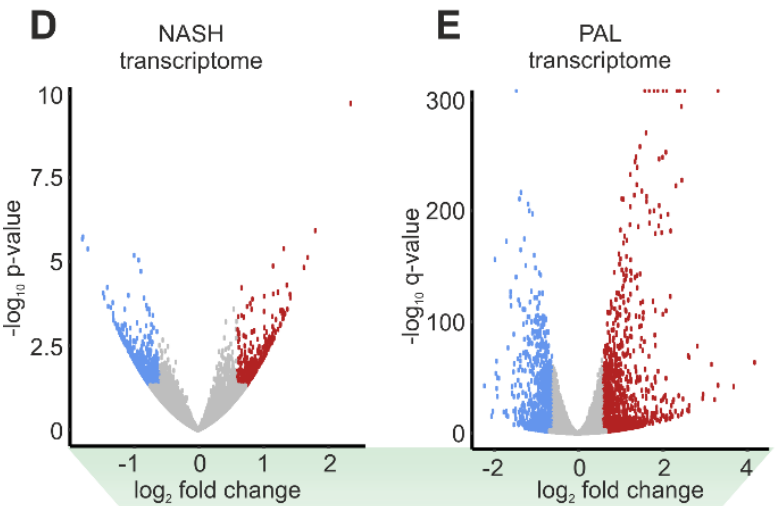

$\mathbf{F}$
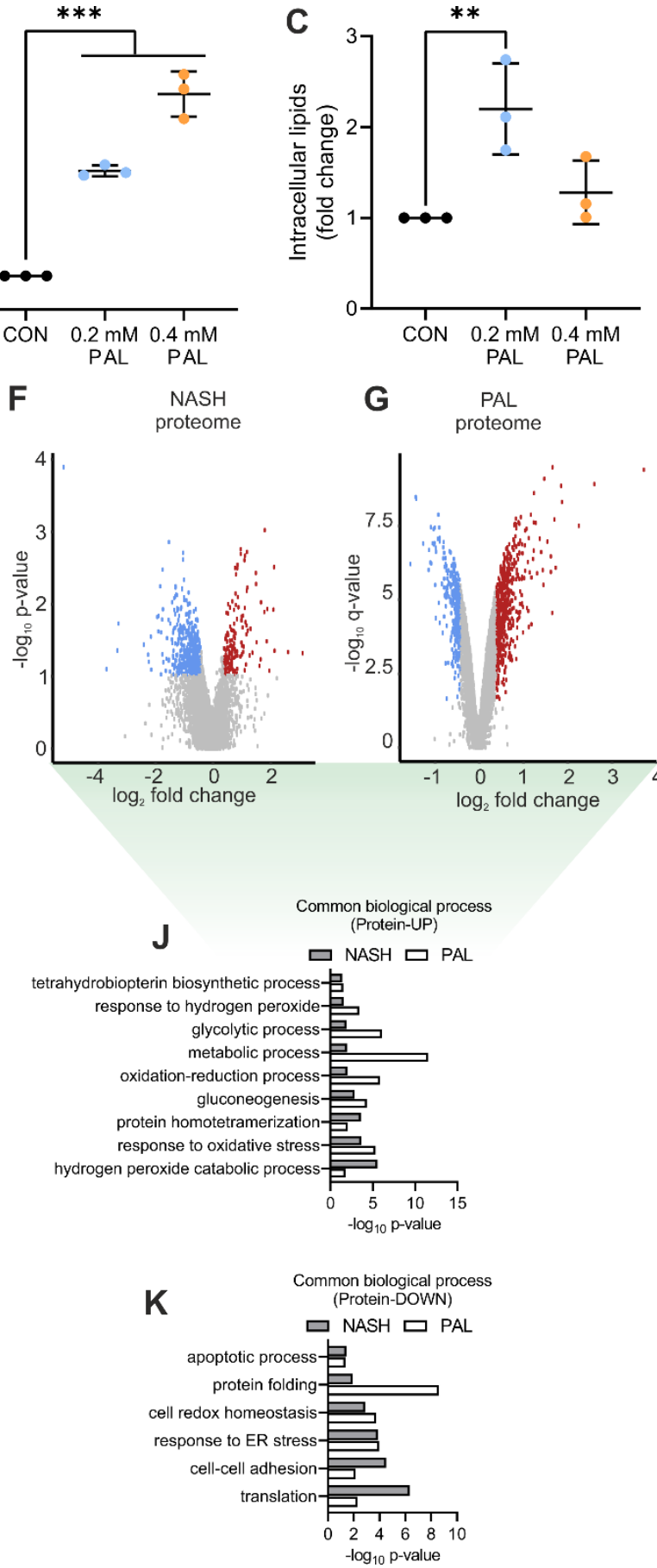

Figure 1. Common molecular adaptations in NASH and lipotoxicity. (A) Characteristics of human subjects. (B and C) Mouse hepatocytes were stimulated with BSA (control) or PAL for $24 \mathrm{~h}$, following which (B) cytotoxicity and (C) intracellular lipid content were measured $(\mathrm{n}=3$ independent experiments, each performed in triplicate; values are mean $\pm \mathrm{SD} ; * * \mathrm{p}<0.01$, $* * * \mathrm{p}<0.001)$. (D-G) Volcano plots showing differentially regulated (D and E) genes and (F 

made available under aCC-BY-NC-ND 4.0 International license.

and $\mathrm{G}$ ) proteins in human liver from NASH patients ( $\mathrm{n}=4$ per group) and mouse hepatocytes stimulated with PAL for $24 \mathrm{~h}$ (n=3 per group). Blue and red dots denote significantly downand up-regulated genes or proteins, respectively. (H-K) Gene ontology analysis showing biological processes commonly regulated in human NASH and mouse hepatocyte lipotoxicity at the ( $\mathrm{H}$ and $\mathrm{I}) \mathrm{RNA}$ and ( $\mathrm{J}$ and $\mathrm{K}$ ) protein level. 
A

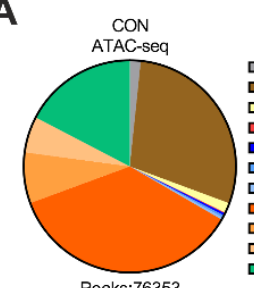

Peaks:76353

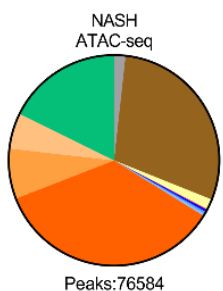

D
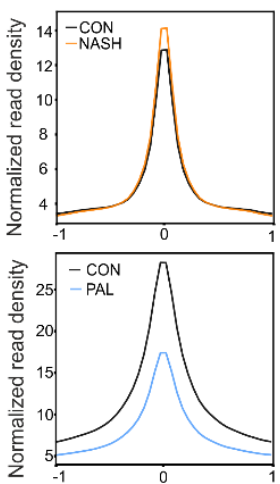

G

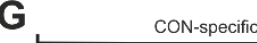

chr5:122,075,334-122,079,026 (3.7kb) chr1:75,723,239-75,727,298 (4kb)
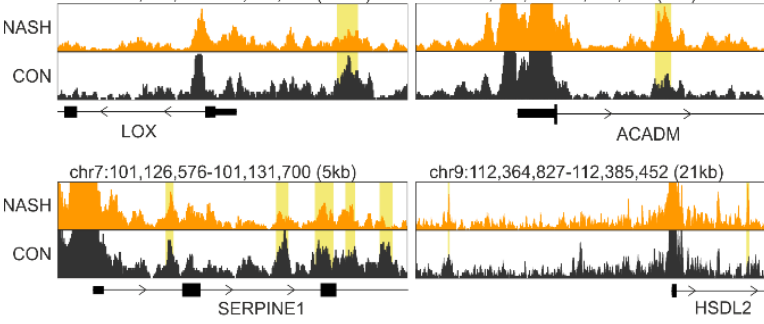

$1.75 \%$ Exon

민. $1.59 \%$ 3' UTR

$0.35 \%$ Downstream (<1 kb)

믈 $35.27 \%$ Promstream $(2-3 \mathrm{~kb})$

$7.71 \%$ Promoter $(1-2 \mathrm{~kb})$

$5.48 \%$ Promoter $(2-3 \mathrm{~kb})$
$17.71 \%$ Distal Intergenic
B
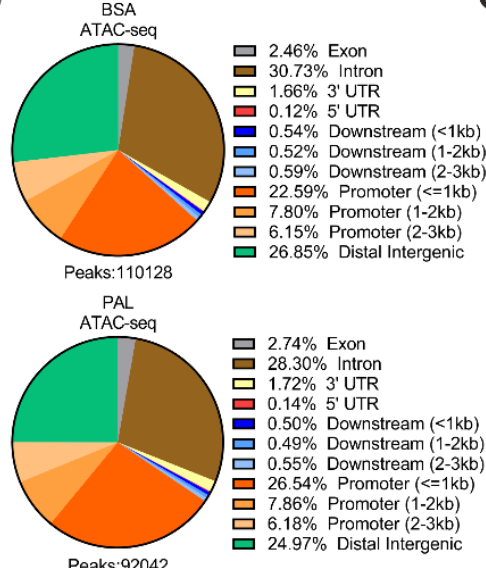

E

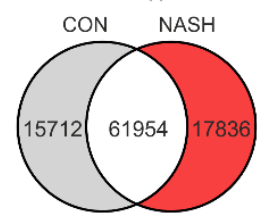

F ATAC-seq peaks

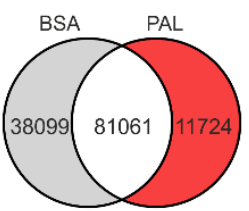

ㅁ $2.46 \%$ Exon

믈 $30.73 \%$ Intron

$0.12 \%$ 5' UTR

$0.54 \%$ Downstream $(<1 \mathrm{~kb})$

$0.59 \%$ Downstream (2-3kb)

口 $22.59 \%$ Promoter $(<=1 \mathrm{~kb})$

ㅁ. $7.80 \%$ Promoter $(1-2 \mathrm{~kb})$

$6.15 \%$ Promoter $(2-3 \mathrm{~kb})$

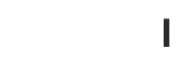

\section{| Biological processes}

(ATAC-seq and RNA-seq)

$\square$ CON-specific
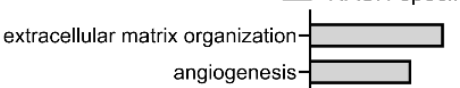

regulation of cell growth-

protein complex assembly

peptidyl-serine phosphorylation

oxidation-reduction process

vitamin metabolic process

cellular response to drug

cellular amino acid metabolic process-

sodium ion transport-

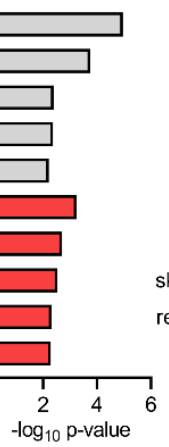

C Normalized ATAC-seq signal

H
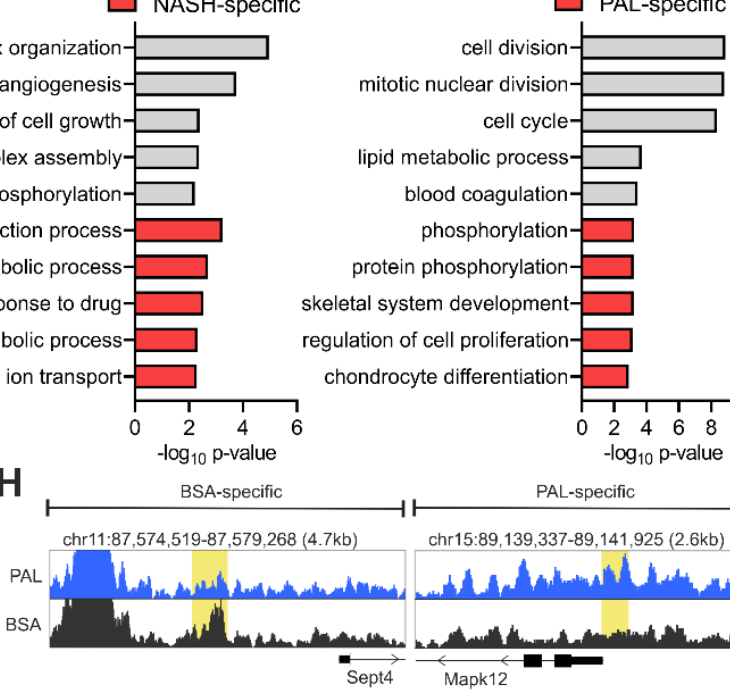

lipid metabolic process-

blood coagulation-

phosphorylation-

protein phosphorylation- $\square$

skeletal system developmentregulation of cell proliferation-

chondrocyte differentiation-

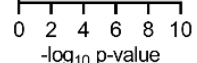

PAL-specific

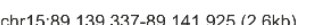

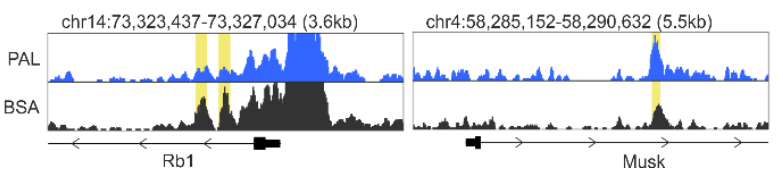

Figure 2. Chromatin accessibility is altered by NASH and lipotoxicity. (A and B)

Annotation of ATAC-seq peaks in (A) human liver ( $\mathrm{n}=4$ per group) and (B) mouse hepatocytes ( $\mathrm{n}=3$ per group). (C) Heat maps of ATAC-seq peaks aligned to their centre $\pm 1 \mathrm{~kb}$. (D) Density plots showing averaged normalized signal of ATAC-seq peaks aligned to their centre $\pm 1 \mathrm{~kb}$. (E and F) Overlap of ATAC-seq peaks between (E) CON and NASH liver or (F) BSA and PAL hepatocytes. (G and $\mathrm{H}$ ) Genome browser views of representative $(\mathrm{G}) \mathrm{CON}$ - or NASH-specific peak in human liver and (H) BSA- or PAL-specific peaks in mouse hepatocytes. (I and J) Gene 
bioRxiv preprint doi: https://doi.org/10.1101/2021 03.24.436772; this version posted March 24, 2021. The copyright holder for this preprint (which was not certified by peer review) is the author/funder, who has granted bioRxiv a license to display the preprint in perpetuity. It is made available under aCC-BY-NC-ND 4.0 International license.

ontology analysis showing biological processes regulated by genes linked to (I) CON- or NASH-specific peak in human liver and (J) BSA- or PAL-specific peaks in mouse hepatocytes. 
A

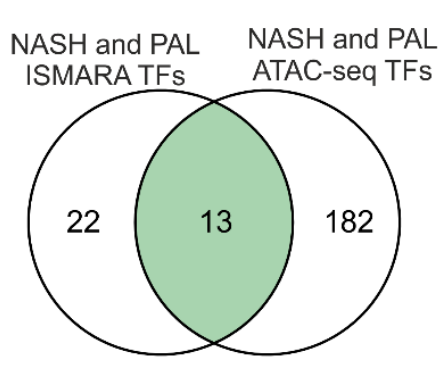

B
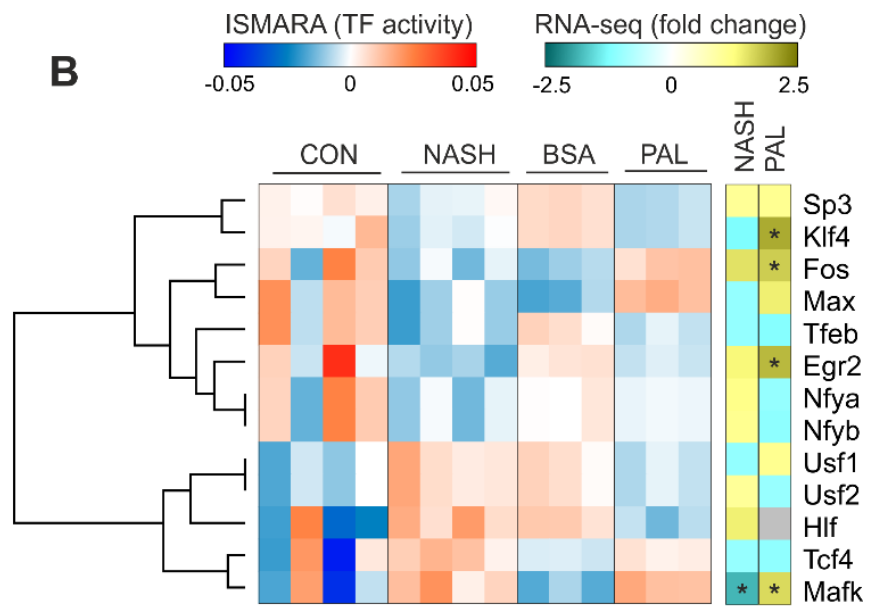

C

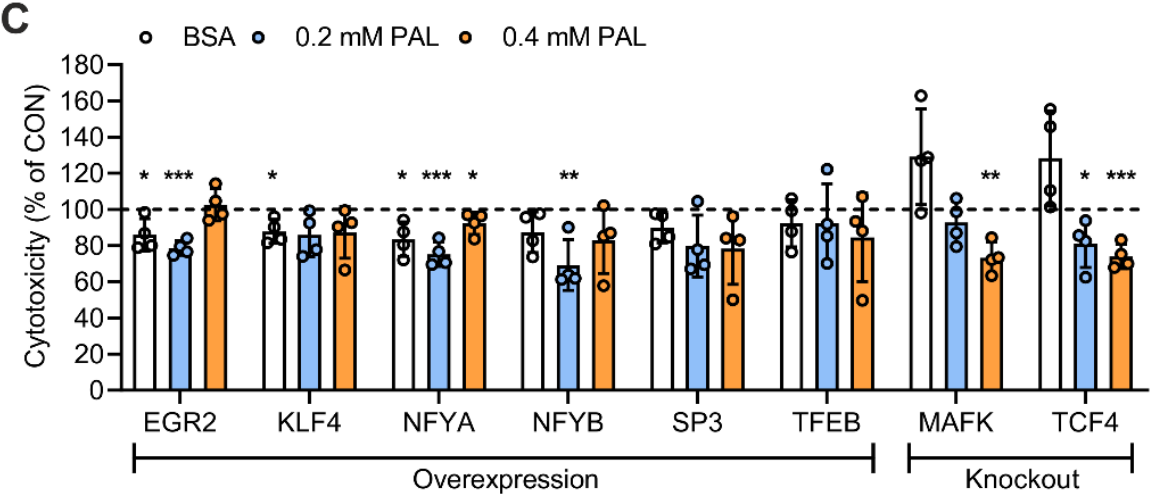

D
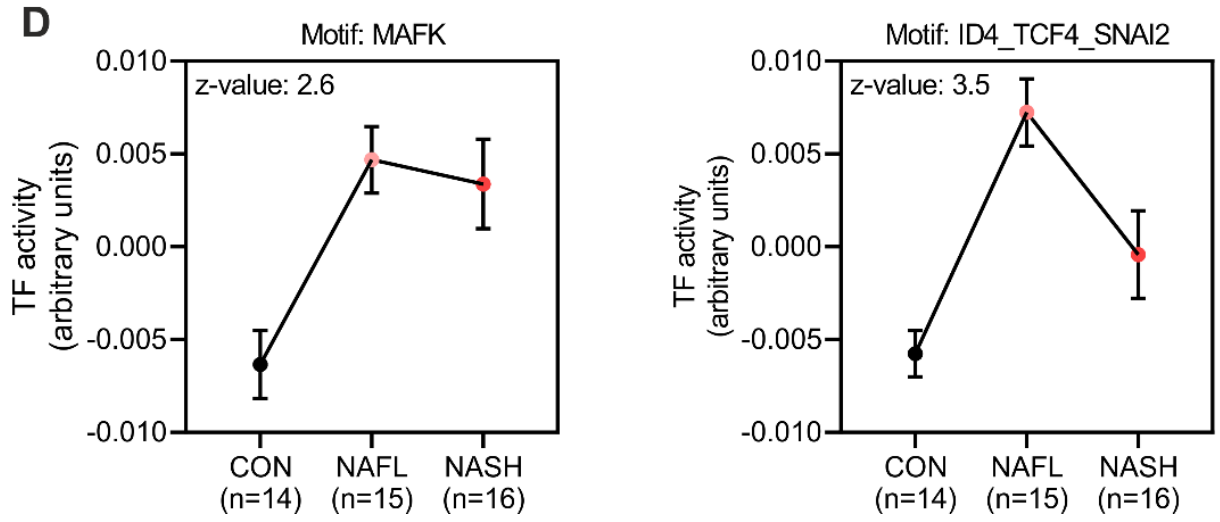

Figure 3. Omics data integration revealed lipotoxicity-sensitive transcription factors. (A)

Overlap between ISMARA (RNA-seq) and motif enrichment analysis (ATAC-seq) predicted transcription factors (TFs). (B) Heat map with ISMARA-predicted activity (left panel; clustering based on predicted TF activity) and gene expression changes (right panel; asterisk denotes a significant change) of commonly regulated TFs. (C) Cytotoxicity measurement following BSA or PAL stimulation for $24 \mathrm{~h}$ in mouse hepatocytes with either overexpression (OE) or knockout $(\mathrm{KO})$ of candidate TFs. Data is expressed as percentage of control cells 
(CON; corresponding to $100 \%$ denoted by the horizontal dashed line) undergoing the same treatment $(n=4$ independent experiments, each performed in triplicate; values are mean $\pm S D$; $* \mathrm{p}<0.05, * * \mathrm{p}<0.01$ and $* * * \mathrm{p}<0.001$ ). (D) ISMARA-predicted activity of MAFK and TCF4 in human liver from CON subjects or NAFL and NASH patients (GEO accession: GSE126848). 

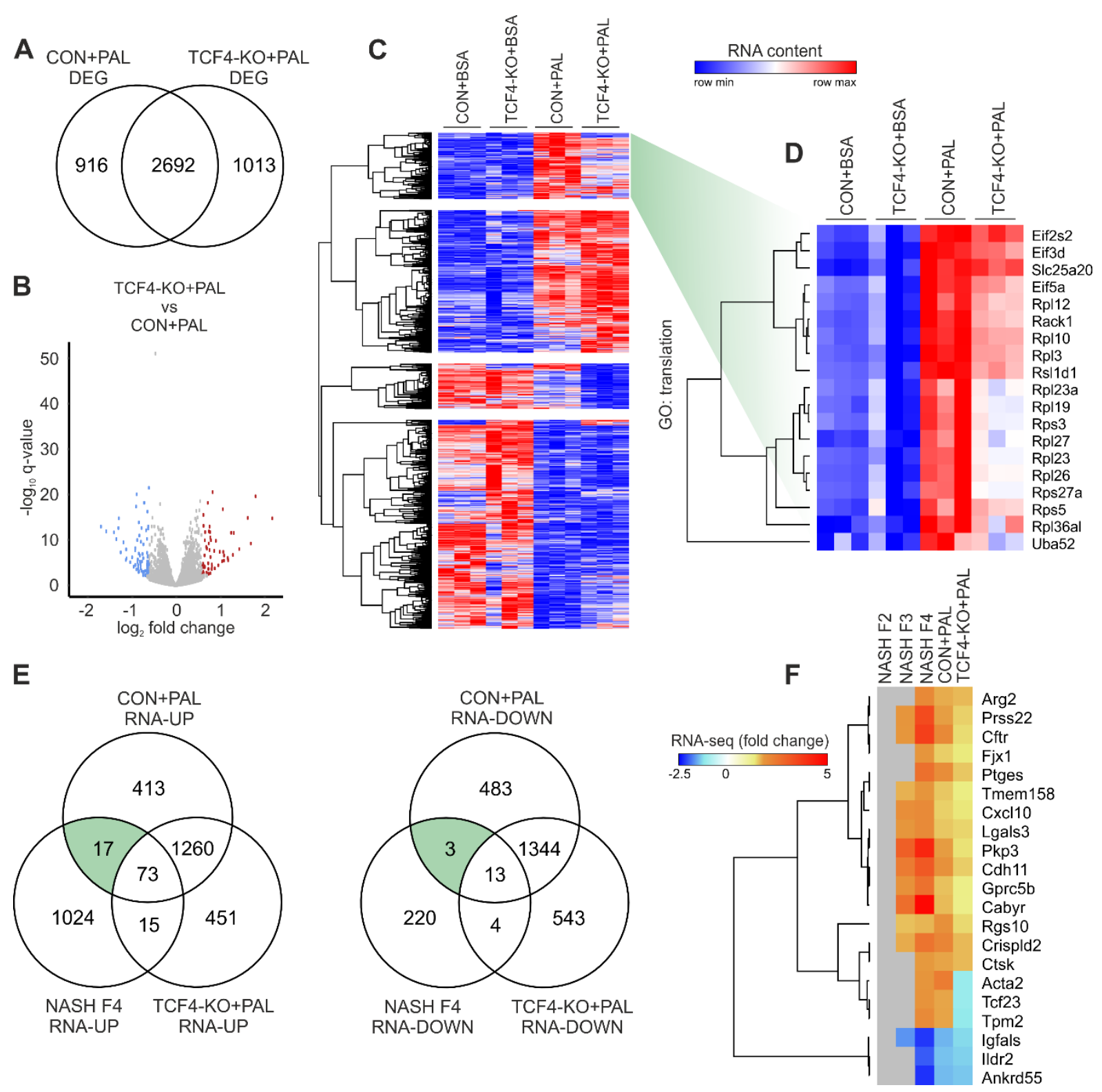

Figure 4. TCF4 target genes are linked to human liver fibrosis in NASH. (A) Overlap between DEG of CON and TCF4-KO hepatocytes stimulated with PAL for $24 \mathrm{~h}(\mathrm{n}=3$ per group). (B) Volcano plot showing DEG when CON and TCF4-KO hepatocytes stimulated with PAL are directly compared (blue and red dots denote significantly down- and up-regulated genes, respectively). (C and D) Heat map and clustering analysis of (C) all TCF4-sensitive genes and (D) genes comprised in the GO term "translation" from cluster 1 analysis. (E) Overlap between DEG in human liver from NASH patients with severe fibrosis (F4; GEO accession: GSE135251) and PAL-stimulate CON or TCF4-KO mouse hepatocytes. (F) Heat 

made available under aCC-BY-NC-ND 4.0 International license.

map and clustering analysis of fold changes of TCF4-sensitive genes commonly regulated in human NASH F4 and mouse hepatocyte lipotoxicity, including data from human liver from NASH patients with mild (F2) and advanced (F3) fibrosis (grey denotes genes not significantly regulated in human NASH). 

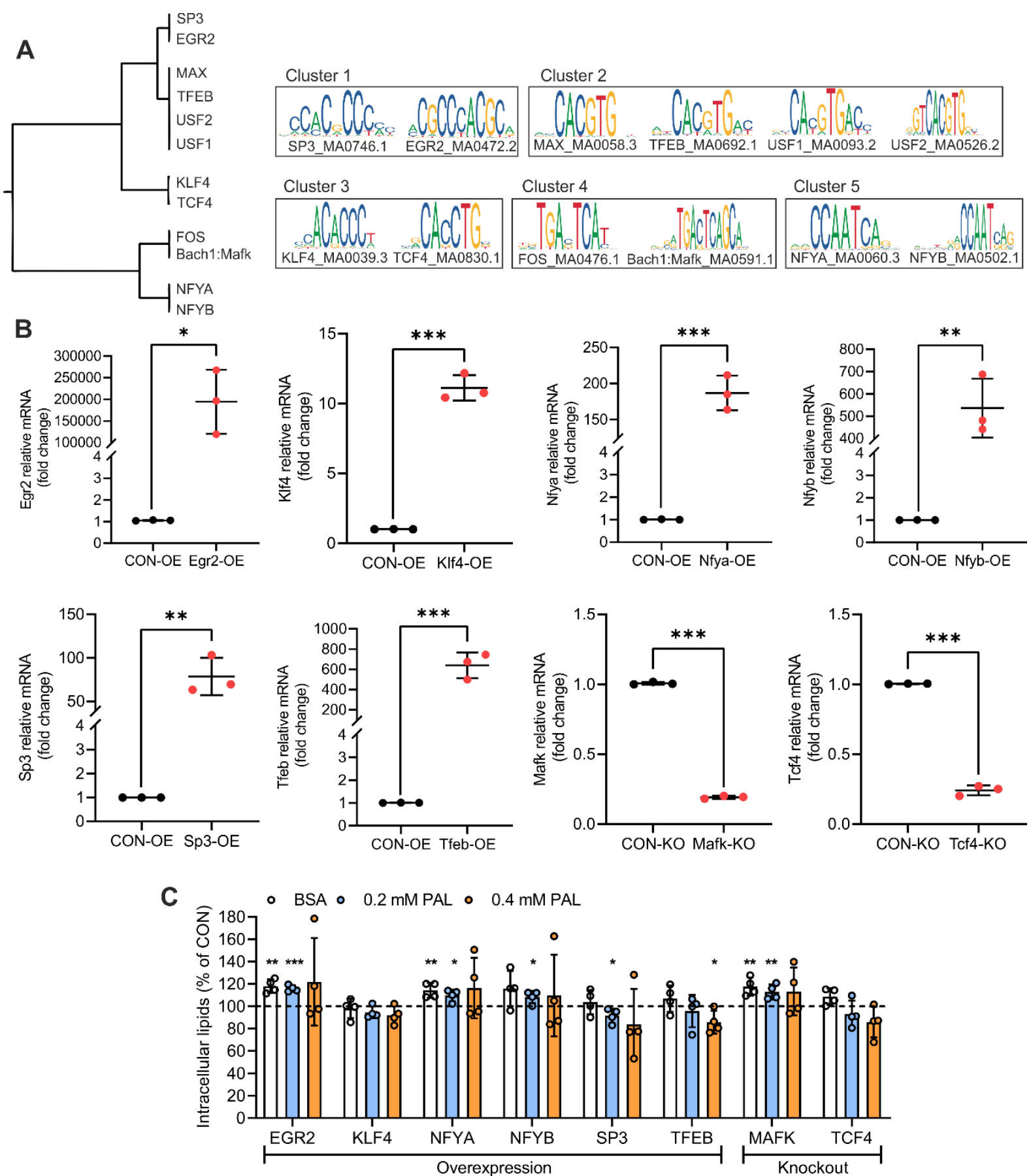

Figure S1. Lipotoxicity-sensitive transcription factors. (A) Transcription factor clustering analysis based on motif consensus sequence (left panel), with the motif logos comprised in the different clusters (right panel). (B) Transcript level of candidate transcription factors following overexpression $(\mathrm{OE})$ or knockout $(\mathrm{KO})$ in mouse hepatocytes compared to their corresponding controls $(\mathrm{CON} ; \mathrm{n}=3$ independent experiments, each performed in triplicate; values are mean $\pm \mathrm{SD} ;{ }^{*} \mathrm{p}<0.05,{ }^{* *} \mathrm{p}<0.01$ and $\left.{ }^{* * *} \mathrm{p}<0.001\right)$. (C) Intracellular lipid content measured 
following BSA or PAL stimulation for $24 \mathrm{~h}$ in mouse hepatocytes with either OE or KO of candidate TFs. Data is expressed as percentage of control cells (CON; corresponding to $100 \%$ denoted by the horizontal dashed line) undergoing the same treatment $(n=4$ independent experiments, each performed in triplicate; values are mean $\pm \mathrm{SD} ;{ }^{*} \mathrm{p}<0.05,{ }^{*} \mathrm{p}<0.01$ and $* * * p<0.001)$ 

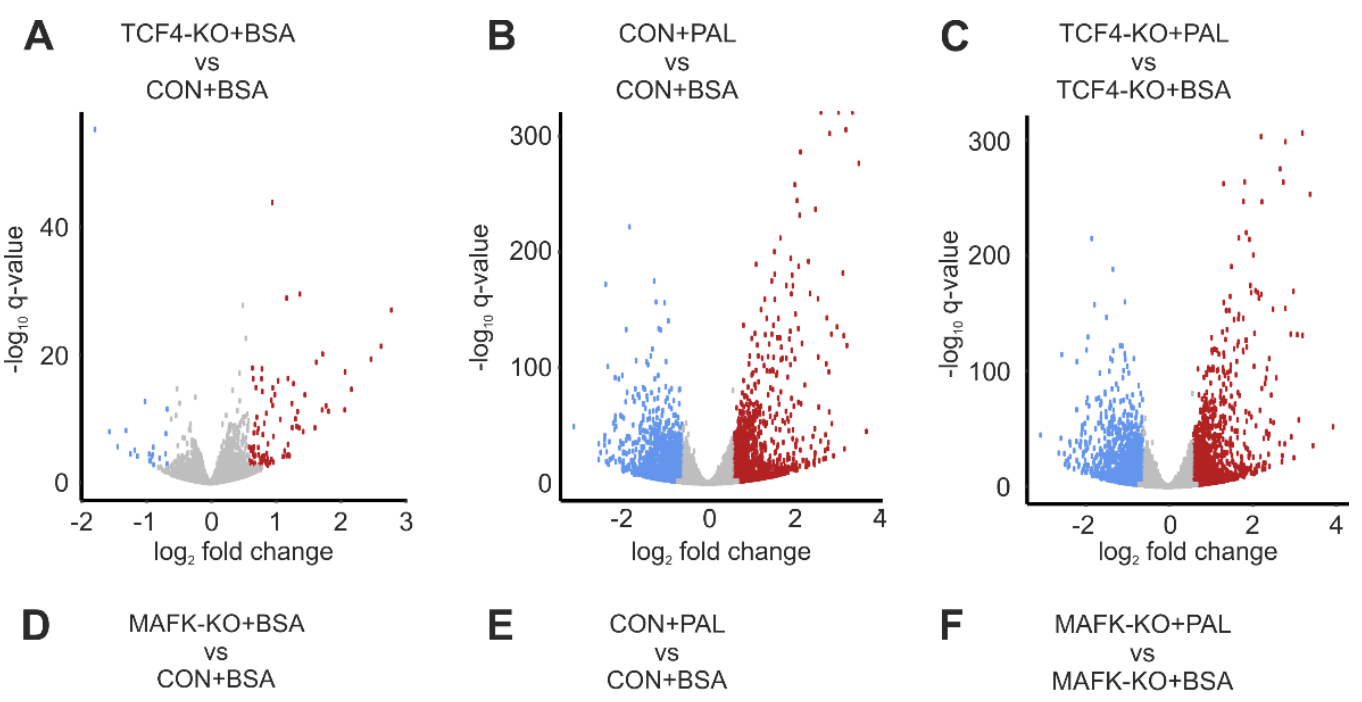

F MAFK-KO+PAL
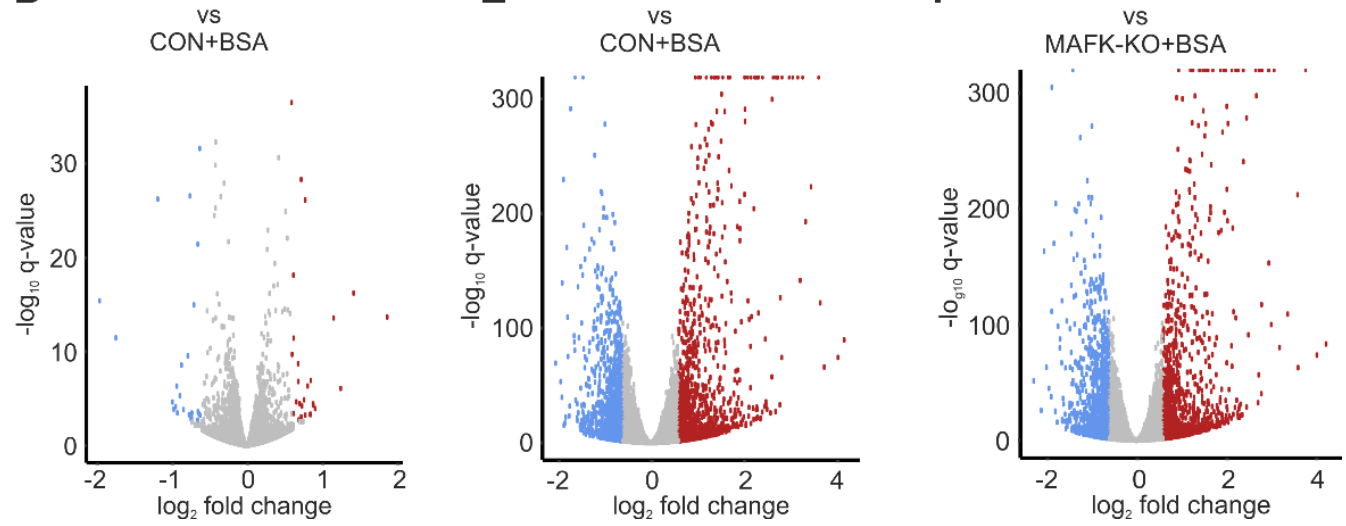

Figure S2. Transcriptomic analysis of MAFK and TCF4 knockout cells. (A-F) Volcano plots showing DEG under basal conditions and following PAL stimulation for $24 \mathrm{~h}$ in (A-C) TCF4-KO or (D-F) MAFK-KO mouse hepatocytes ( $\mathrm{n}=3$ per group; CON: control; blue and red dots denote significantly down- and up-regulated genes, respectively). 


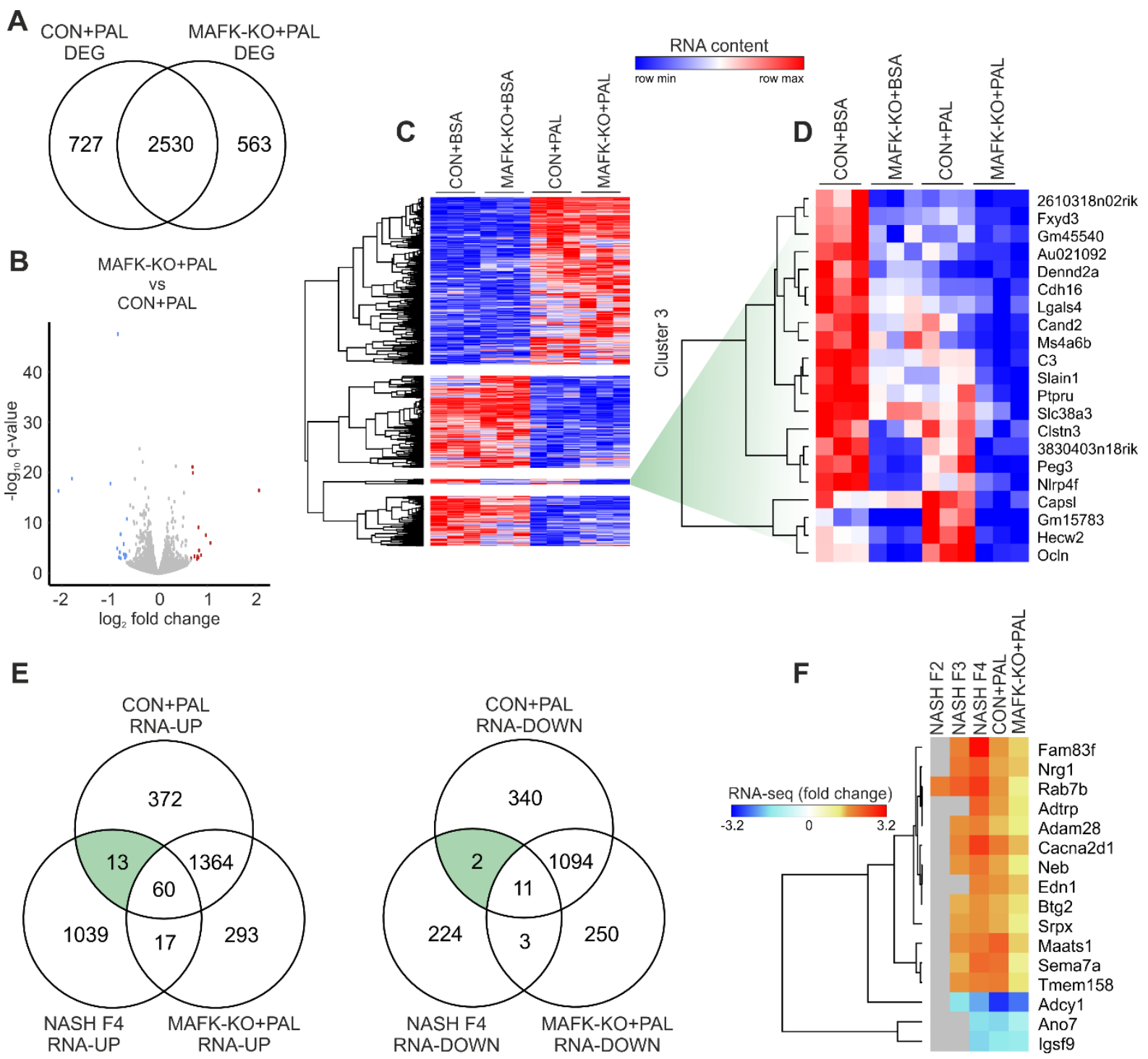

Figure S3. MAFK transcriptional network in lipotoxicity and NASH. (A) Overlap between

DEG of CON and MAFK-KO hepatocytes stimulated with PAL for $24 \mathrm{~h}$ ( $\mathrm{n}=3$ per group). (B)

Volcano plot showing DEG when CON and MAFK-KO hepatocytes stimulated with PAL are directly compared (blue and red dots denote significantly down- and up-regulated genes, respectively). (C and D) Heat map and clustering analysis of (C) all MAFK-sensitive genes and (D) genes comprised in cluster 3. (E) Overlap between DEG in human liver from NASH patients with severe fibrosis (F4; GEO accession: GSE135251) and PAL-stimulate CON or MAFK-KO mouse hepatocytes. (F) Heat map and clustering analysis of fold changes of MAFK-sensitive genes commonly regulated in human NASH F4 and mouse hepatocyte 
bioRxiv preprint doi: https://doi.org/10.1101/2021.03.24.436772; this version posted March 24, 2021. The copyright holder for this preprint (which was not certified by peer review) is the author/funder, who has granted bioRxiv a license to display the preprint in perpetuity. It is made available under aCC-BY-NC-ND 4.0 International license.

lipotoxicity, including data from human liver from NASH patients with mild (F2) and advanced

(F3) fibrosis (grey denotes genes not significantly regulated in human NASH). 


\section{REFERENCES}

Ahrne, E., Glatter, T., Vigano, C., Schubert, C., Nigg, E.A., and Schmidt, A. (2016). Evaluation and Improvement of Quantification Accuracy in Isobaric Mass Tag-Based Protein Quantification Experiments. J Proteome Res 15, 2537-2547.

Al Attar, A., Antaramian, A., and Noureddin, M. (2021). Review of galectin-3 inhibitors in the treatment of nonalcoholic steatohepatitis. Expert Rev Clin Pharmacol, 1-8.

Ao, N., Ma, Z., Yang, J., Jin, S., Zhang, K., Luo, E., and Du, J. (2020). Liraglutide ameliorates lipotoxicity-induced inflammation through the mTORC1 signalling pathway. Peptides 133 , 170375.

Appenzeller-Herzog, C., and Hall, M.N. (2012). Bidirectional crosstalk between endoplasmic reticulum stress and mTOR signaling. Trends Cell Biol 22, 274-282.

Bailey, T.L., and Machanick, P. (2012). Inferring direct DNA binding from ChIP-seq. Nucleic acids research 40 , e128.

Balwierz, P.J., Pachkov, M., Arnold, P., Gruber, A.J., Zavolan, M., and van Nimwegen, E. (2014). ISMARA: automated modeling of genomic signals as a democracy of regulatory motifs. Genome Res 24, 869-884.

Cazanave, S., Podtelezhnikov, A., Jensen, K., Seneshaw, M., Kumar, D.P., Min, H.K., Santhekadur, P.K., Banini, B., Mauro, A.G., A, M.O., et al. (2017). The Transcriptomic Signature Of Disease Development And Progression Of Nonalcoholic Fatty Liver Disease. Sci Rep 7, 17193.

Chen, Y., Griffiths, A., Wang, J., Zhang, T., Song, Q., and Song, Z. (2020). Inositol-requiring enzyme 1alpha links palmitate-induced mTOR activation and lipotoxicity in hepatocytes. American journal of physiology. Cell physiology 319, C1130-C1140.

Corces, M.R., Trevino, A.E., Hamilton, E.G., Greenside, P.G., Sinnott-Armstrong, N.A., Vesuna, S., Satpathy, A.T., Rubin, A.J., Montine, K.S., Wu, B., et al. (2017). An improved 
ATAC-seq protocol reduces background and enables interrogation of frozen tissues. Nat Methods 14, 959-962.

De Minicis, S., Rychlicki, C., Agostinelli, L., Saccomanno, S., Trozzi, L., Candelaresi, C., Bataller, R., Millan, C., Brenner, D.A., Vivarelli, M., et al. (2013). Semaphorin 7A contributes to TGF-beta-mediated liver fibrogenesis. Am J Pathol 183, 820-830.

Freese, N.H., Norris, D.C., and Loraine, A.E. (2016). Integrated genome browser: visual analytics platform for genomics. Bioinformatics 32, 2089-2095.

Friedman, S.L., Neuschwander-Tetri, B.A., Rinella, M., and Sanyal, A.J. (2018). Mechanisms of NAFLD development and therapeutic strategies. Nat Med 24, 908-922.

Govaere, O., Cockell, S., Tiniakos, D., Queen, R., Younes, R., Vacca, M., Alexander, L., Ravaioli, F., Palmer, J., Petta, S., et al. (2020). Transcriptomic profiling across the nonalcoholic fatty liver disease spectrum reveals gene signatures for steatohepatitis and fibrosis. Sci Transl Med 12.

Guri, Y., Colombi, M., Dazert, E., Hindupur, S.K., Roszik, J., Moes, S., Jenoe, P., Heim, M.H., Riezman, I., Riezman, H., et al. (2017). mTORC2 Promotes Tumorigenesis via Lipid Synthesis. Cancer Cell 32, 807-823 e812.

Haberle, V., and Stark, A. (2018). Eukaryotic core promoters and the functional basis of transcription initiation. Nature reviews. Molecular cell biology 19, 621-637.

Herat, L., Rudnicka, C., Okada, Y., Mochizuki, S., Schlaich, M., and Matthews, V. (2017). The Metalloproteinase ADAM28 Promotes Metabolic Dysfunction in Mice. Int J Mol Sci 18.

Hetz, C., Zhang, K., and Kaufman, R.J. (2020). Mechanisms, regulation and functions of the unfolded protein response. Nature reviews. Molecular cell biology 21, 421-438.

Iacobini, C., Menini, S., Ricci, C., Blasetti Fantauzzi, C., Scipioni, A., Salvi, L., Cordone, S., Delucchi, F., Serino, M., Federici, M., et al. (2011). Galectin-3 ablation protects mice from diet-induced NASH: a major scavenging role for galectin-3 in liver. J Hepatol 54, 975-983. 
Im, Y.R., Hunter, H., de Gracia Hahn, D., Duret, A., Cheah, Q., Dong, J., Fairey, M., Hjalmarsson, C., Li, A., Lim, H.K., et al. (2020). A systematic analysis of rodent models implicates adipogenesis and innate immunity in pathogenesis of fatty liver disease. bioRxiv, 2020.2012.2015.422799.

Jeftic, I., Jovicic, N., Pantic, J., Arsenijevic, N., Lukic, M.L., and Pejnovic, N. (2015). Galectin3 Ablation Enhances Liver Steatosis, but Attenuates Inflammation and IL-33-Dependent Fibrosis in Obesogenic Mouse Model of Nonalcoholic Steatohepatitis. Mol Med 21, 453-465. Leung, A., Parks, B.W., Du, J., Trac, C., Setten, R., Chen, Y., Brown, K., Lusis, A.J., Natarajan, R., and Schones, D.E. (2014). Open chromatin profiling in mice livers reveals unique chromatin variations induced by high fat diet. The Journal of biological chemistry 289, 2355723567.

Leung, A., Trac, C., Du, J., Natarajan, R., and Schones, D.E. (2016). Persistent Chromatin Modifications Induced by High Fat Diet. The Journal of biological chemistry 291, 1044610455.

Listenberger, L.L., Han, X., Lewis, S.E., Cases, S., Farese, R.V., Jr., Ory, D.S., and Schaffer, J.E. (2003). Triglyceride accumulation protects against fatty acid-induced lipotoxicity. Proceedings of the National Academy of Sciences of the United States of America 100, 30773082 .

Liu, G.Y., and Sabatini, D.M. (2020). mTOR at the nexus of nutrition, growth, ageing and disease. Nature reviews. Molecular cell biology 21, 183-203.

Mahony, S., and Benos, P.V. (2007). STAMP: a web tool for exploring DNA-binding motif similarities. Nucleic acids research 35, W253-258.

Pedroza, M., To, S., Smith, J., and Agarwal, S.K. (2019). Cadherin-11 contributes to liver fibrosis induced by carbon tetrachloride. PloS one 14, e0218971. 
Ruan, W., Pan, R., Shen, X., Nie, Y., and Wu, Y. (2019). CDH11 promotes liver fibrosis via activation of hepatic stellate cells. Biochem Biophys Res Commun 508, 543-549.

Santhekadur, P.K., Kumar, D.P., and Sanyal, A.J. (2018). Preclinical models of non-alcoholic fatty liver disease. J Hepatol 68, 230-237.

Siersbaek, M., Varticovski, L., Yang, S., Baek, S., Nielsen, R., Mandrup, S., Hager, G.L., Chung, J.H., and Grontved, L. (2017). High fat diet-induced changes of mouse hepatic transcription and enhancer activity can be reversed by subsequent weight loss. Sci Rep 7, 40220.

Suppli, M.P., Rigbolt, K.T.G., Veidal, S.S., Heeboll, S., Eriksen, P.L., Demant, M., Bagger, J.I., Nielsen, J.C., Oro, D., Thrane, S.W., et al. (2019). Hepatic transcriptome signatures in patients with varying degrees of nonalcoholic fatty liver disease compared with healthy normal-weight individuals. Am J Physiol Gastrointest Liver Physiol 316, G462-G472.

Suzuki, A., and Diehl, A.M. (2017). Nonalcoholic Steatohepatitis. Annu Rev Med 68, 85-98. Vuppalanchi, R., Noureddin, M., Alkhouri, N., and Sanyal, A.J. (2021). Therapeutic pipeline in nonalcoholic steatohepatitis. Nat Rev Gastroenterol Hepatol.

Wang, Y., Viscarra, J., Kim, S.J., and Sul, H.S. (2015). Transcriptional regulation of hepatic lipogenesis. Nature reviews. Molecular cell biology 16, 678-689.

Wang, Y., Yang, F., Gritsenko, M.A., Wang, Y., Clauss, T., Liu, T., Shen, Y., Monroe, M.E., Lopez-Ferrer, D., Reno, T., et al. (2011). Reversed-phase chromatography with multiple fraction concatenation strategy for proteome profiling of human MCF10A cells. Proteomics 11, 2019-2026.

Xiao, Y., Kim, M., and Lazar, M.A. (2020). Nuclear receptors and transcriptional regulation in non-alcoholic fatty liver disease. Mol Metab, 101119. 
Zhang, X., Shen, J., Man, K., Chu, E.S., Yau, T.O., Sung, J.C., Go, M.Y., Deng, J., Lu, L., Wong, V.W., et al. (2014). CXCL10 plays a key role as an inflammatory mediator and a noninvasive biomarker of non-alcoholic steatohepatitis. J Hepatol 61, 1365-1375. 